\title{
A Language of Tactile Motion Instructions
}

\author{
Daniel Spelmezan \\ RWTH Aachen University \\ 52056 Aachen, Germany \\ spelmezan@cs.rwth- \\ aachen.de
}

\author{
Anke Hilgers \\ RWTH Aachen University \\ 52056 Aachen, Germany \\ anke.hilgers@rwth- \\ aachen.de
}

\author{
Jan Borchers \\ RWTH Aachen University \\ 52056 Aachen, Germany \\ borchers@cs.rwth- \\ aachen.de
}

\begin{abstract}
Tactile motion instructions are vibrotactile feedback patterns delivered across the entire body that indicate how to move during physical activities. This work investigates the perception and identification of such patterns, based on two different metaphors, under stationary and active situations. We further combine and sequentially trigger different patterns to explore whether tactile motion instructions are understandable as a simple language. A tactile language could represent motion sequences to guide students during demanding exercises. Finally, the presented studies provide insights into perception and interpretation of tactile feedback and help to inform a design space for full-body vibrotactile cues.
\end{abstract}

\section{Categories and Subject Descriptors}

H.5.2 [Information Interfaces and Presentation]: User Interfaces-Haptic $\mathrm{I} / \mathrm{O}$

\section{General Terms}

Design, Experimentation, Human Factors

\section{Keywords}

Vibrotactile feedback, tactile language, real-time instructions, physical activities, sports training, motor skill learning.

\section{INTRODUCTION}

To master sports techniques, students need to learn appropriate timing and correct execution of various body movements. For example, to accurately perform turns while snowboarding, riders should move their weight to the forward pointing foot, turn their upper body towards the intended riding direction, and redistribute their weight evenly between both feet after pivoting the board. Such physical movements, however, can be difficult to perform especially by students unfamiliar to new and quickly changing motion sequences. Moreover, beginners who descend the slope focus on keeping their balance and oftentimes forget important parts in the required motion sequence. Similar situations occur, for example, in dancing. Dancers need to coordinate and to appropriately time their movements both to the music and to their partner.

Copyright is held by the author/owner(s). MobileHCI 2009, September 15 - 18, 2009, Bonn, Germany. ACM 978-1-60558-281-8.
To teach correct technique and to help beginners get the feel of the motion, coaches typically use demonstrations, spoken instructions, and even physical guidance by touching and placing the student's body into the appropriate position and desired movement at the preferred speed. In sports that take place in noisy outdoor environments, however, audio feedback is less appropriate. In winter or water sports, for example, instructors also cannot give real-time feedback and physical guidance because they are spatially separated from their students. In such cases, tactile motion instructions are an alternative technique for providing hints for corrections during physical activities [6]. Vibrotactile patterns triggered at key body locations represent specific movements and signal how to adjust posture, such as turn your upper body to the right, shift your weight to the left foot, or flex your legs.

This work focuses on improving the discriminability of tactile motion instructions and investigates how well patterns are perceived and interpreted when combined similarly to words in a language; sequentially triggered instructions that remind students of correct order and timing of a sequence of movements involved in an exercise or in physical activities.

\section{RELATED WORK}

Vibrotactile feedback delivered across the body has been applied to pedestrian navigation [3], to translate written text to tactile cues [4], or to increase spatial awareness during flights [7]. Tactons [2] represent structured, abstract messages that convey information non-visually. Tactile feedback has also been explored to improve gesture interaction [1].

\section{STUDY OVERVIEW}

Our previous study conducted under stationary and active situations revealed that participants most often misinterpreted tactile motion instructions delivered around the waist, which represented torso rotation to the left or right [6]. Though these patterns were always perceived during physical activities, participants could not reliably differentiate the direction rendered around the waist. Results further indicated that certain instructions can be based either on a push or a pull metaphor: tactile cues delivered laterally to the right side of the body can either evoke the sensation of being pushed to the left or pulled to the right. Likewise, cues delivered to the back or chest can be interpreted either as lean upper body forward or backward. These metaphors for encoding instructions seem to be a matter of user preference and could influence performance in quickly responding to cues under mobile tasks. The following studies address these questions: 


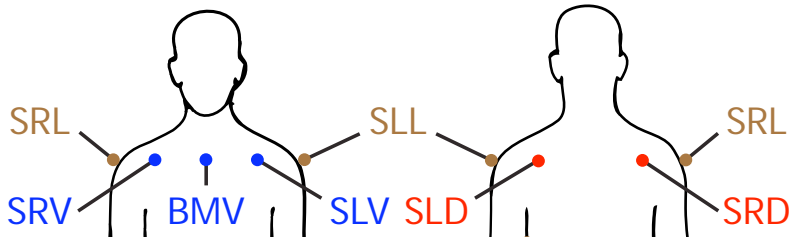

Figure 1. Actuator placement around the shoulders with three-letter acronyms (Body/Shoulder, Left/Medial/Right, Dorsal/Lateral/Ventral).

1. Study 1 aimed at finding alternative and well discriminable patterns for representing upper body rotation.

2. Study 2 investigated the influence of two metaphors in identifying patterns under active situations. Motion instructions were further combined and sequentially triggered to assess if patterns allow for building a practicable language that guides students during physical activities.

\section{STUDY 1: IMPROVING PATTERN DISCRIMINABILITY}

This study aimed at finding alternative body locations and cues for encoding the instructions turn upper body to the left $(T L)$ vs. turn upper body to the right $(T R)$. Based on previous results [6] and an additional brainstorming session, we composed patterns that exploit the sensory saltation phenomenon [5] to convey directional information to the skin:

- Saltation around one shoulder

- Compound saltation around both shoulders

- Horizontal saltation at the upper chest

Fig. 1 illustrates motor placement on the torso using Nokia 3210 vibration motors inserted into thin plastic tubes. Cues were delivered with full intensity (on/off) at $5 \mathrm{~V}$. In order to experiment with different sensations on the skin, we varied the pattern length by applying tactile cues that consisted either of a single brief pulse $P$ of $100 \mathrm{~ms}$ duration per motor or of three sequential pulses $P^{3}$ per motor with $50 \mathrm{~ms}$ pause between stimuli [6]. Table 1 summarizes eight potential patterns for signaling upper body rotation to the left using the notation introduced in [6]. Patterns delivered to only one shoulder were displayed in two directions, starting either at the chest and concluding at the back or vice versa ( $T L_{1}$ to $\left.T L_{4}\right)$. Compound saltation around both shoulders rendered two tactile patterns sequentially $\left(T L_{5}, T L_{6}, T L_{7}\right)$.

\subsection{Experimental Setup}

Ten university students aged 21-27 (average 24.1 years, seven male) participated. Subjects stood upright and were first presented with all patterns to become familiar with artificial vibrotactile feedback. During the experiment, two patterns that both represented either turn left or turn right were randomly triggered with a delay of three seconds. Every possible pair was triggered twice. The order in which pairs were triggered was counterbalanced. A forced-choice paradigm was applied for answers: participants were asked in which direction they would turn and which pattern they preferred for indicating turn your upper body to the left (right).
Table 1. Eight alternative patterns intended for turn left. When played back in reversed order, these patterns could represent turn right $T R_{x}$.

\begin{tabular}{ccc} 
Pattern & sec. & Tactile cues \\
\hline$T L_{1}$ & 0.4 & $P(S L V) \rightarrow P(S L L) \rightarrow P(S L D)$ \\
$T L_{2}$ & 0.4 & $P(S R D) \rightarrow P(S R L) \rightarrow P(S R V)$ \\
$T L_{3}$ & 1.3 & $P^{3}(S L V) \rightarrow P^{3}(S L L) \rightarrow P^{3}(S L D)$ \\
$T L_{4}$ & 1.3 & $P^{3}(S R D) \rightarrow P^{3}(S R L) \rightarrow P^{3}(S R V)$ \\
$T L_{5}$ & 0.85 & $T L_{1} \rightarrow T L_{2}$ \\
$T L_{6}$ & 2.65 & $T L_{3} \rightarrow T L_{4}$ \\
$T L_{7}$ & 1.75 & $T L_{5} \rightarrow T L_{5}$ \\
$T L_{8}$ & 1.3 & $P^{3}(S R V) \rightarrow P^{3}(B M V) \rightarrow P^{3}(S L V)$ \\
\hline
\end{tabular}

\subsection{Results}

All participants followed directional information rendered on the skin to decide in which direction to turn. For example, $T L_{2}$ was interpreted as turn left, though this pattern was evoked around the right shoulder, started at the back, and concluded at the chest. Two-tailed Wilcoxon signed ranks tests yielded a significant difference between $T L_{5}(82.5 \%)$ and $T L_{7}$ and between $T R_{1}(82.5 \%)$ and $T R_{8}(T=0 ; n=$ $9 ; p<0.05)$; Our volunteers further tended to prefer vibration around both shoulders $(62.5 \%)$ over vibration around one shoulder $(37.5 \%)$ and patterns with single motor pulses $P(62.5 \%)$ over triple pulses $P^{3}(37.5 \%)$.

Debriefing after delivering patterns revealed that six participants interpreted $T L_{5}, T L_{6}$, and $T L_{7}$ as request to turn the whole body around $180^{\circ}$ or $360^{\circ}$. These patterns delivered cues sequentially to both shoulders. Cues triggered only at one shoulder rather represented less extensive movements, such as a turn of the upper body around the spine. Three participants stated that patterns with three pulses per motor felt slower and thus seemed to request slower turns compared to the shorter patterns that triggered one single pulse per motor.

\subsection{Discussion}

Our participants preferred single rotation around both shoulders over double rotation and vibration around one shoulder over directional information rendered at the chest. This indicates that patterns $T L_{7}, T R_{7}, T L_{8}$, and $T R_{8}$ can be dismissed. Though sequential rotation around both shoulders, such as $T L_{5}$ and $T L_{6}$, was slightly preferred over vibration around one shoulder, we assume that these patterns might nevertheless cause confusion under mobile tasks; similar to the original instructions delivered around the waist [6], these patterns use the same actuators as patterns $T R_{5}$ and $T R_{6}$ and can only be reliably discriminated by perceiving the rendered direction. For the following study, we thus decided to use $T L_{3}, T R_{3}, T L_{4}$ and $T R_{4}$ around one shoulder to signal body rotation. These patterns further have the same duration as other instructions presented in [6]. However, patterns $T L_{1}, T R_{1}, T L_{2}$ and $T R_{2}$ can as well represent rotation and might be preferred to indicate more rapid turns.

\section{STUDY 2: COMBINED MOTION INSTRUCTIONS}

This study investigated the influence of the push and the pull encoding metaphors on pattern identification accuracy 
Table 2. The basic set of motion instructions [6]. $R_{X}$ denotes saltation (rabbit patterns) upwards, downwards, or horizontally on the body.

\begin{tabular}{lcc} 
Instruction & Acronym & Sensation \\
\hline Stretch the legs & $S L$ & $R_{U}$ \\
Flex the legs & $F L$ & $R_{D}$ \\
Shift weight to the left foot & $W L$ & $R_{U}$ \\
Shift weight to the right foot & $W R$ & $R_{U}$ \\
Lean upper body to the left & $L L$ & $R_{U}$ \\
Lean upper body to the right & $L R$ & $R_{U}$ \\
Lean upper body forward & $L F$ & $P^{3}$ \\
Lean backward (straighten up) & $L B$ & $P^{3}$ \\
Turn upper body to the left & $T L$ & $R_{H}$ \\
Turn upper body to the right & $T R$ & $R_{H}$ \\
\hline
\end{tabular}

during relaxed and active situations. Different motion instructions were additionally combined and triggered sequentially to assess how well participants perceive and identify extended instructions. We used the Nintendo Wii Fit balance board for active situations and asked participants to play Snowboard Slalom. This game is physically and cognitively demanding and requires to pass between flags on the screen.

\subsection{Experimental Setup}

20 university students participated (aged 22-29, average 24.6 years, 16 male). All volunteers stated to regularly perform some sports. Two participants were snowboarders, two had previously tried out the balance board but not the snowboarding game. None of our participants had previously experienced artificial vibrotactile feedback from vibrating motors.

Participants were equipped with 30 actuators across their body, which were sewn onto tightly fitting cycling shirts and trousers (see [6] for actuator placement in addition to Fig. 1). Table 2 summarizes the basic set of tactile motion instructions that represents ten movements. Before the experiment, these instructions were triggered once to allow participants experience how vibration feels on their body.

A forced-choice study was conducted to determine if our candidates intuitively prefer to move towards or away from cues delivered to the chest, to the back, or laterally to the thighs/torso. For example, upon perceiving pulses laterally at the right thigh, participants were asked to state whether they would intuitively respond with $W L$ (push) or with $W R$ (pull). These results determined the encoding metaphor for the basic instruction set that participants had to subsequently learn and respond to during the final experiment.

We divided participants into two groups (intuitive, counterintuitive). For the intuitive group, instructions were encoded according to a participant's preferred metaphor. Instructions for the counter-intuitive group were encoded to represent a participant's counter-intuitive metaphor. The pull metaphor required to move towards vibration: Cues delivered laterally to the right (left) thigh represented $W R(W L)$, pulses to the back (chest) represented $L B(L F)$, saltation at the back (front) of thighs signaled $F L(S L)$. Saltation around the left (right) shoulder indicated $T L(T R)$, encoded by $T L_{3}$ $\left(T R_{4}\right)$. In contrast, the push metaphor required to move away from vibration, such as $W L(W R)$ for cues delivered laterally to the right (left) thigh, or $L F$ ( $L B)$ for pulses triggered at the back (chest). $T L_{4}$ delivered around the right shoulder signaled turn left, whereas $T R_{3}$ delivered around the left shoulder signaled turn right. During a subsequent ten minutes training session, all participants learned their customized basic instruction set by pressing buttons on a GUI, which triggered tactile cues. After this training session, our volunteers further tried out the balance board to become familiar with controlling the snowboarder on the screen.

The final experiment tested perception and interpretation of instructions while balancing on the board and while being relaxed (stationary), which we regard as baseline for optimal perception and interpretation of instructions. During the relaxed situation, participants stood still and listened to soft music over headphones that blocked auditory cues from vibrating motors. While balancing on the board, participants heard the sound of the game played back over loudspeakers. The order of relaxed and active situations was counterbalanced across participants to avoid learning effects. We informed participants that they would experience basic and additionally extended instructions that consisted of two sequentially triggered patterns. These combined instructions were not learned during initial training and addressed lower and upper body movements $(W L \rightarrow T L, W L \rightarrow T R$, $W R \rightarrow T L, W R \rightarrow T R$ ), exclusively upper body movements $(L L \rightarrow T L, L L \rightarrow T R, L R \rightarrow T L, L R \rightarrow T R)$, and exclusively lower body movements $(W L \rightarrow F L, W L \rightarrow$ $S L, W R \rightarrow F L, W R \rightarrow S L)$. In total 22 instructions were tested (10 basic, 12 combined). Instructions were randomly triggered twice during both conditions with 10-15 seconds delay after answers. The participants' task was to respond to motion instructions by first saying out aloud the perceived instructions and then by performing the learned movements.

\subsection{Results}

Nine participants preferred the push metaphor. Under relaxed condition, performance of the intuitive group in identifying basic instructions was $99.5 \%$ and $100 \%$ for combined instructions. The counter-intuitive group achieved overall scores of $96.5 \%$ and $95.4 \%$. The intuitive group performed significantly better for combined instructions that exclusively addressed lower-body patterns ( $100 \%$ vs. $88.8 \%$, paired $\mathrm{t}(3)$ $=0.009, \mathrm{p}=0.05)$ : the counter-intuitive group misinterpreted once the 1 st pattern and seven times the 2 nd pattern.

During the active situation, participants did not respond to nine instructions. The intuitive group misinterpreted 33 times the 1st pattern and nine times the 2nd pattern of combined instructions, the counter-intuitive group misinterpreted eight times the 1st pattern and ten times the 2nd pattern. Fig. 2 shows performance in identifying basic instructions (intuitive $97.5 \%$, counter-intuitive $97 \%$ ), Fig. 3 performance in identifying combined instructions $(83.8 \%$ vs. $93.3 \%)$. The intuitive group performed significantly worse at combined instructions addressing exclusively upper body (87.5\% vs. 


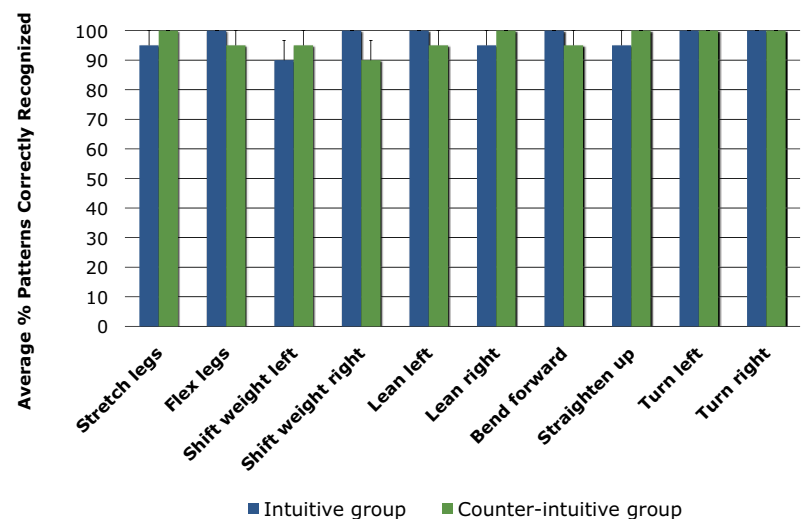

Figure 2. Correct scores of basic instructions (with standard error).

$97.5 \%$, paired $\mathrm{t}(3)=0.02, \mathrm{p}=0.05)$ or lower body movements $(78.8 \%$ vs. $91.3 \%$, paired $\mathrm{t}(3)=0.04, \mathrm{p}=0.05)$.

Participants mentioned no difficulties in learning counterintuitive instructions, though more training might be beneficial. Some participants stated they liked tactile cues, which made the game more fun to play. Patterns that indicated turn left/right using vibration around the shoulders were clear to follow. One former ballet dancer was reminded of dance lessons and imagined tactile cues might work well to teach dancing. However, having to speak while balancing on the board made the game more difficult to play. Few participants answered only on request. Others tended to mix up directions: though they knew what patterns actually meant, they responded with left instead of right (or vice versa).

\subsection{Discussion}

Compared to [6] the new patterns that indicate turn left/right were recognized with near perfect accuracy. In identifying the ten basic instructions under active situations, both groups achieved overall correct scores almost identical to the baseline for optimal perception and interpretation of cues measured while stationary. These results suggest that the counterintuitive metaphor does not significantly degrade learning and interpretation of basic instructions.

However, participants in the intuitive group performed significantly worse in responding to sequentially triggered instructions on the balance board; they misinterpreted eight times more often the first pattern of combined instructions. One reason for this degraded performance might be that the intuitive group had probably paid less attention to accurately verbalize novel extended cues, since these cues were regarded as being intuitive. Future studies should measure participants' responses differently than through speech, since responding verbally is delayed, requires additional cognitive load, and might cause participants to mix up directions. The fact that instructions were triggered randomly and thus could occur at times that did not match to the currently performed movements during the game might have further negatively skewed our results, such as an instruction that indicated to turn left though the participant's intention was to turn to the right.

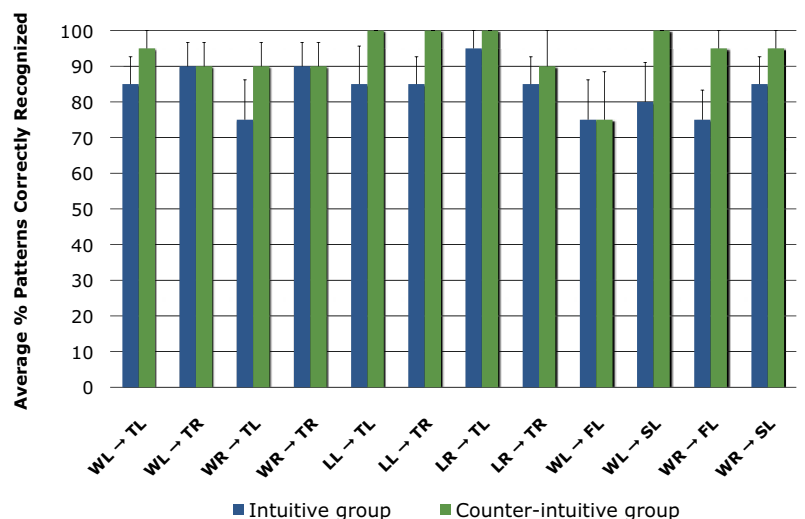

Figure 3. Correct scores of extended instructions (with standard error, the higher error rates account for the increased probability to misinterpret at least one of the two sequentially triggered patterns).

\section{CONCLUSIONS}

This work investigated perception and interpretation of fullbody tactile motion instructions based on two different encoding metaphors. The results suggest that the presented set of tactile patterns is well perceived and interpreted under active situations and might be used similarly to a spoken language to guide students during physical activities. Future work should explore alternative patterns for creating a design space of vibrotactile cues and investigate the potential benefits of tactile feedback to teach new motor skills.

\section{ACKNOWLEDGMENTS}

This work was partly funded by the German B-IT Foundation.

8. REFERENCES

1. Ahmaniemi, T., Lantz, V., and Marila, J. Dynamic Audiotactile Feedback in Gesture Interaction. Proc. MobileHCI 2008, ACM, 339-342.

2. Brewster, S., and Brown, L. M. Tactons: Structured Tactile Messages for Non-Visual Information Display. Proc. AUIC 2004, Australian Computer Society, 15-23.

3. Ertan, S., Lee, C., Willets, A., Tan, H., and Pentland, A. A Wearable Haptic Navigation Guidance System. Proc. ISWC 1998, IEEE, Washington, 164-165.

4. Geldard, F.A. Cutaneous Coding of Optical Signals: The Optohapt. Perception \& Psychophysics 1, 1966, 377-381.

5. Geldard, F.A. Sensory Saltation: Metastability in the Perceptual World. Lawrence Erlbaum Associates, Hillsdale, N.J., USA, 1975.

6. Spelmezan, D., Jacobs, M., Hilgers, A., and Borchers, J. Tactile Motion Instructions for Physical Activities. Proc. CHI 2009, ACM, New York, 2243-2252.

7. Van Veen, H. A. H. C. and van Erp, J. B. F. Tactile Information Presentation in the Cockpit. Proc. Haptic Human-Computer Interaction, LCNS 2058, Springer (2001), 174-181. 